\title{
Towards predicting process parameters for selective laser melting of titanium alloys through the modelling of melt pool characteristics
}

\author{
T van Rhijn ${ }^{1, a}$, W du Preez ${ }^{2, b}$, M Maringa ${ }^{1, c}$, D Kouprianoff ${ }^{2, d}$ \\ ${ }^{1}$ Department of Mechanical and Mechatronics Engineering, Faculty of Engineering, Built Environment and Information Technology, Central \\ University of Technology, Free State, South Africa \\ ${ }^{2}$ Centre for Rapid Prototyping and Manufacturing, Faculty of Engineering, Built Environment and Information Technology, Central \\ University of Technology, Free State, South Africa \\ Email: ${ }^{a}$ thinusvr611@gmail.com, ${ }^{b}$ wdupreez@cut.ac.za, ${ }^{c}$ mmaringa@cut.ac.za, ${ }^{d}$ dkouprianoff@cut.ac.za
}

\begin{abstract}
Various researchers have investigated the use of experimental melt pool characterization to speed up the optimisation process of selective laser melting parameters and found this to be possible. From their studies, it has become clear that the incorporation of modelling into this approach could provide efficient and predictable results that would minimise the experimental validation work required. This paper reports on progress made towards characterising the melt pool through simulation. The development of a numerical model is discussed. Subsequently, experimental validation of the numerical model is presented. This is done through a comparison of the simulation results with the experimentally determined cross-sectional geometry of single tracks created with various sets of process parameters. Melt pool size and shape are considered. Based on these results, it is concluded that using an identical simulation setup, verified simulation method, and verified material properties, it was possible to accurately determine the melt pool geometry for some, but not all process parameters.
\end{abstract}

Keywords: Selective laser melting, melt pool characteristics, Ti6A14V, modelling, process parameter optimisation

\section{Introduction}

The selective laser melting (SLM) process involves a large number of process variables, with many of these being interdependent. This makes the process of fully optimising the process parameters for new materials challenging, as well as expensive and timeconsuming (Sun, et al., 2017). The reason for this is the extensive experimental work required to produce statistically convincing data for proving the repeatability and reliability of the process (Nicoletto, et al., 2018]). Therefore, a novel approach such as modelling is needed to reduce the experimental work required for the optimisation process.

Gong, et al. (2014) attempted to rapidly optimise process parameters by characterising cross-sectional melt pool dimensions and geometry. Their study concluded that the characterisation of the melt pool provided helpful information in determining ideal process parameters. In particular, it was seen that the geometry of the melt pool could be used to accurately predict the optimal hatch spacing distance. Lastly, and of greatest importance to this study, it was concluded that the incorporation of simulation work could predict process parameters with greater efficiency. Recently, multiple new numerical models have been created (Rauniyar \& Chou, 2019) (Majeed, et al., 2019) (Ridolfi, et al., 2019). With the accuracy of these models continuously improving, the possibility of using numerical modelling to predict process parameters becomes a greater possibility. This paper forms part of a broader study that is attempting to achieve the above-mentioned. The setup of a numerical model of an SLM single track on a bare Ti6Al4V plate with no powder and the experimental validation thereof are presented and discussed.

\section{Material and methods}

For the validation of the model, a systematic approach was used. Firstly, the complexity of the model was reduced as much as possible. This was done by removing the powder layer from the substrate and not considering multiple layers or tracks. Consequently, the SLM process was reduced to the movement of a laser along a bare metal surface in a straight line (single track). This removed the added complexity of powder layer thickness, hatch spacing, particle spreading and laser absorptivity inherent to a powder bed.

When considering single tracks in SLM, there are only two userinput parameters that greatly influence the melt pool dimensions. These are laser power and scanning speed. To validate a numerical model that will be used to determine unknown melt pool geometries at various process parameters, the accuracy of the model at the extremes of these parameters needed to be investigated. Therefore, it was decided to simulate four distinctly different melt pool phenomena with widely different process parameters. The first of these was weak penetration, which can be identified by partial melting of the powder layer. Secondly, an optimal process resulting in a uniform melt pool was considered. The third phenomenon that was considered was balling, which results in separation of the melt pool due to high scanning speeds (Gibson, et al., 2015) Lastly, the keyhole phenomenon (King, et al., 2014), caused by a too high laser energy density, was evaluated. The process parameters used 
in this study to simulate the above-mentioned phenomena are given in Table I.

Table I: Various parameter sets used for modelling

\begin{tabular}{lcc}
\hline Parameter set & $\begin{array}{c}\text { Laser power } \\
\text { (W) }\end{array}$ & $\begin{array}{c}\text { Scanning speed } \\
(\mathbf{m} / \mathbf{s})\end{array}$ \\
\hline Weak penetration & 80 & 1.2 \\
$\begin{array}{l}\text { Optimal (Yadroitsava, et al., } \\
\text { 2015) }\end{array}$ & 170 & 1.2 \\
Balling & 350 & 2.0 \\
Keyhole melting & 340 & 0.6 \\
\hline
\end{tabular}

\subsection{Numerical model}

The numerical model was developed using the additive manufacturing (AM) package of the commercial software FLOW3D (FLOW-3D, 2021). This software was used to apply a finite volume method (FVM) model to determine the cross-sectional width and depth of a melt pool created by the SLM process.

FLOW-3D numerically solves the equations of mass-, momentumand energy-conservation. The free surface of the melt pool is tracked by utilising the volume-of-fluid (VOF) technique. More detailed explanations of the equations and techniques can be found in papers utilising the same software (Cheng, et al., 2019) (Wu, et al., 2017). This approach enables the accurate simulation of the geometry of the melt pool, as well as the interface of the free surface with the surrounding air. The simulation was set up assuming incompressible flow of the melt.

A simulation domain with width $\mathrm{x}$ breadth $\mathrm{x}$ depth of $2400 \times 760$ x $395 \mu \mathrm{m}$ was used for all but one simulation. As the keyhole parameters lead to a deeper melt pool, a depth of $495 \mu \mathrm{m}$ was used for this simulation. A track length of $1600 \mu \mathrm{m}$ was simulated for all process parameters to ensure that steady-state was reached within the simulation. The top boundary was set as a constant pressure outflow, and all other boundaries were defined as walls at room temperature $(293 \mathrm{~K})$. This most closely represents physical reality.

After conducting a study of mesh dependency, it was found that using a cell size of $5 \mu \mathrm{m}$ for the smaller area in which the melt pool formed, and a larger cell size of $20 \mu \mathrm{m}$ in the surrounding areas to

Table II: Material properties used in the model

\begin{tabular}{lcc}
\hline Material property & Value & Sources \\
\hline Convective heat transfer coefficient & $100 \mathrm{~W} / \mathrm{mK}$ & (Cengel \& Ghajar, 2015) \\
Surface tension coefficient & $1.493-4 \times 10^{-4}\left(\mathrm{~T}-\mathrm{T}_{\mathrm{m}}\right)$ & (Egry, et al., 2010) (Mohr, et al., 2020) \\
Density & Temp dep. & (Mills, 2002) (Schmon, et al., 2017) \\
Viscosity & Temp dep. & (Mohr, et al., 2020) (Lu, et al., 2017) \\
Specific heat & Temp dep. & (Boivineau, et al., 2006) (Milosevic \& Aleksic, 2012) \\
Thermal conductivity & Temp dep. & (Mills, 2002)(Mohr, et al., 2020) \\
Saturation temperature & $3315 \mathrm{~K}$ & (Brandes \& Brook, 1992) \\
Latent heat of vaporisation & $9830 \mathrm{~kJ} / \mathrm{kg}$ & (Tan, et al., 2019) \\
Specific heat ratio & 1.67 & (Ford \& Lee, 2001) \\
Vapour specific heat & $695 \mathrm{~J} / \mathrm{kgK}$ & (Brandes \& Brook, 1992) \\
Absorptivity & 0.41 & (Boley, et al., 2016) (Xie, et al., 1997) \\
Emissivity & 0.32 & (Mohr, et al., 2020) \\
\hline
\end{tabular}

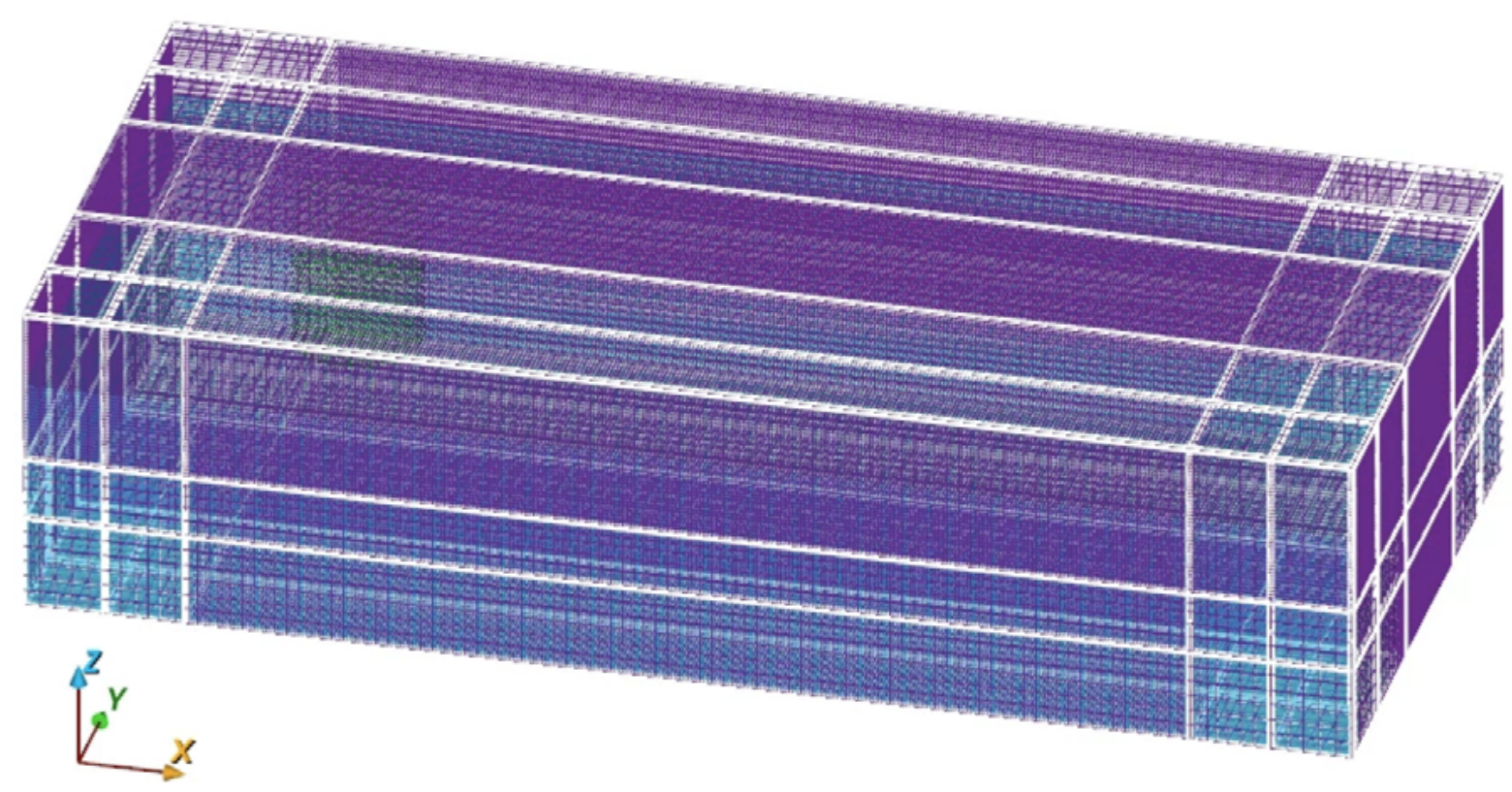

Figure 1: Graphical representation of the simulation domain. The use of larger cell sizes on the edges can be seen here 

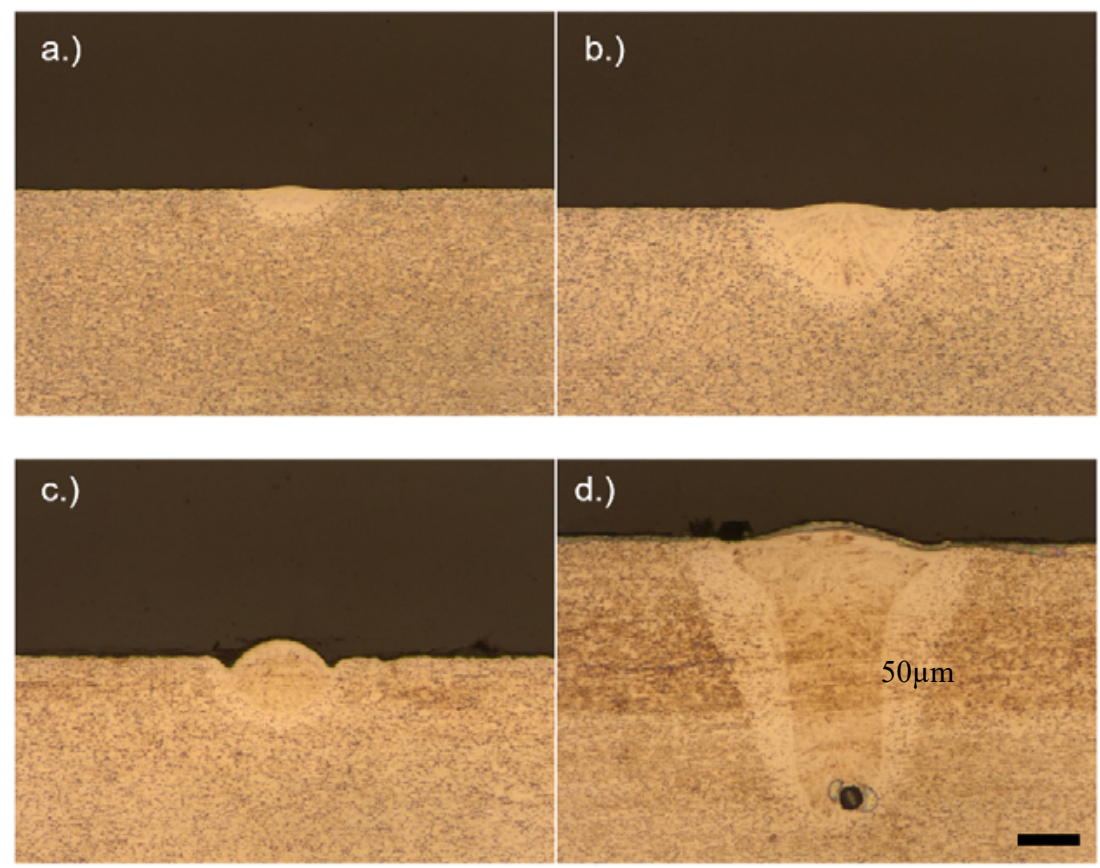

Figure 2: Micrographs of the cross-sections of a.) weak penetration b.) optimal c.) balling and d.) keyhole melting process parameters

account for thermal diffusion would be sufficient. The domain and its meshing can be seen in Figure 1

Physics models activated in the setup included temperaturedependent material properties, volumetric thermal expansion, evaporation, solidification, gravity and all modes of heat transfer. The heat transfer modes included conduction between different phases (solid, liquid and vapour), convective cooling through the air above the bed, as well as radiative cooling to the surroundings. The material property values used in the model, as well as references to the sources in which the values were found, are provided in Table II.

The FLOW-WELD package was used to simulate the laser energy applied to the top free surface of the substrate. The laser used in the experimental work had a Gaussian heat distribution with a spot diameter of $80 \mu \mathrm{m}$. The same spot diameter was used in the setup of the simulation. Laser power and scanning speed for the individual simulations were set according to the parameter sets given in Table I. Multiple Fresnel reflections of the laser were enabled. Tracking of the laser was stopped after $99 \%$ of the laser energy had been absorbed to account for most of the heat applied to the free surface due to the laser.

This setup resulted in a mesh with 2,4 million cells for the keyhole mesh and 1,8 million cells for all other meshes. The simulation times varied substantially due to the different parameter sets and domain sizes used. The total simulation time varied between six hours for the weak penetration parameter set and more than twenty hours for the keyhole parameter set. This was achieved by using a computer with an Intel 10980XE processor with 16 cores and 128 GB of memory.

\subsection{Experimental procedure}

Samples were created with an EOS M280 machine according to the process parameter sets in Table I on a bare Ti6Al4V plate. No specific surface finish was obtained before processing. For each parameter set, fifteen single tracks, each with a length of $20 \mathrm{~mm}$, were created. After the tracks had been created, samples were cut from the plate using wire electrical discharge machining to expose the cross-sections of the molten tracks. After the samples had been cut, they were mounted, polished and etched with Kroll's reagent according to standard sample preparation techniques to expose the microstructure of the samples. From these samples, measurements of the depth and width of all single tracks were recorded using optical microscopy.

Table III: Depth and width of the melt pool for the different parameter sets

\begin{tabular}{lcccc}
\hline Parameter set & Weak penetration & Optimal & Balling & Keyhole \\
\hline Average depth $(\mu \mathrm{m})$ & 23,2 & 75,7 & 66,7 & 152,4 \\
Standard deviation $(\mu \mathrm{m})$ & 1,10 & 1,52 & 2,20 & 67,75 \\
RSD $(\%)$ & 4,74 & 2,01 & 3,29 & 44,44 \\
Average width $(\mu \mathrm{m})$ & 73,9 & 117,9 & 106,2 & 195,0 \\
Standard deviation $(\mu \mathrm{m})$ & 2,32 & 1,65 & 1,52 & 9,65 \\
RSD $(\%)$ & 3,15 & 1,40 & 1,43 & 4,95 \\
\hline
\end{tabular}



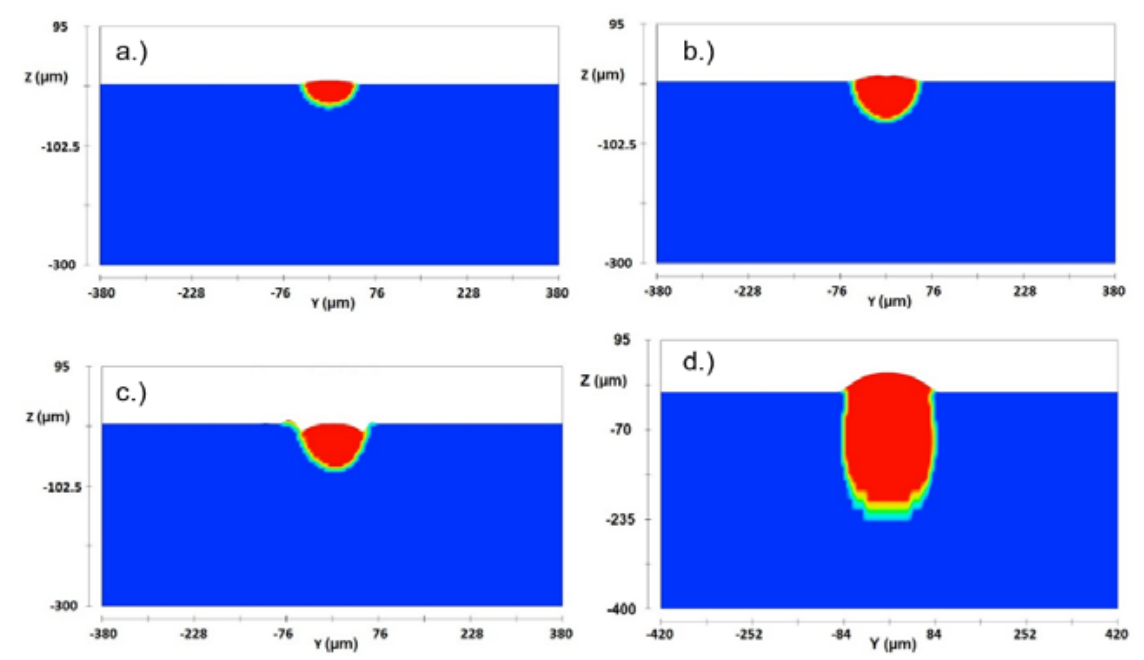

Figure 3: Cross-section results of the simulations of a.) weak penetration process parameters b.) optimal process parameters c.) balling process parameters and d.) keyhole melting process parameters

\section{Results and discussion}

\subsection{Experimental results}

In Figure 1 cross-sectional micrographs from the various process parameter sets can be seen, while Table III shows the depth and width measurements determined from the cross-sectional micrographs.

For most of the parameter sets, the creation of the melt pool was steady, with only small deviations in the cross-sectional melt pool geometry. This is confirmed by the relative standard deviations (RSD) in Table III that are all less than 5\%, except for the keyhole depth. For the keyhole parameter set, however, the depth of the melt pool varied considerably, ranging from $57 \mu \mathrm{m}$ to almost 250 $\mu \mathrm{m}$. Therefore, Figure 1(d) does not represent a typical result for the keyhole mode melting. This figure also shows keyhole induced porosity.

\subsection{Simulation results}

Figure 2 shows the cross-sections of the simulations that were run. The blue colour indicates the metal substrate, and the red colour the molten region. The coloured region between the blue and red regions indicates a region of partial melting.

The variation of the dimensions of the simulated melt pools from the experimentally determined dimensions is shown in Table IV.

\subsubsection{Weak penetration parameter set melt pool}

When comparing the experimental melt pool shape with the simulation result, it is clear that the melt pool depth of the simulation is considerably deeper than the actual depth (Table III and IV). It differs by more than $70 \%$ from the experimentally obtained values. The width of the melt pool compares well, with less than $10 \%$ difference between the simulation and experimental results. It was initially thought that the difference in depth might be due to enhanced conductivity as a result of a too coarse mesh. Subsequent simulations, however, indicated that although reducing the cell size to $3 \mu \mathrm{m}$ did result in the melt pool depth decreasing, it was still more than $60 \%$ deeper than the experimental values. Possible reasons for this discrepancy are discussed in Section 3.3. The latter simulation resulted in a melt pool width of $76 \mu \mathrm{m}$, differing from experimental results by less than four per cent.

\subsubsection{Optimal parameter set melt pool.}

The melt pool dimensions for the optimal process parameters were very close to those for the experimental samples, with dimensions varying by less than three per cent, falling well within two standard deviations of the experimental results. When critically analysing the difference in melt pool geometries, it was found that although the melt pool shapes correlate well, there were still slight variations, such as the difference in the angle of the sidewall of the melt pool, as well as a melt pool that protruded higher above the surface for the simulation.

\subsubsection{Balling parameter set melt pool}

When comparing the melt pools of the balling parameter set, these varied by approximately twenty per cent from the experimental values. The peculiar melt pool shape (protruding upper part) obtained by the balling parameter set can be seen in the simulation shown in Figure 2(c). This result is, however, believed to be by chance, since no other cross-section along the track had this shape. Apart from this, the dimensions of the melt pool along the length of the simulated track remained stable.

Table IV:Simulation dimensions and variation from experimental dimensions for the different process parameter sets

\begin{tabular}{lcccc}
\hline Parameter set & Weak penetration & Optimal & Balling & Keyhole \\
\hline Depth $(\mu \mathrm{m})$ & 40 & 75 & 76 & 259 \\
Variation from experimental $(\%)$ & 72.4 & -0.9 & 13.9 & 70.4 \\
Width $(\mu \mathrm{m})$ & 80 & 115 & 126 & 179 \\
Variation from experimental $(\%)$ & 8,3 & -2.5 & 18.9 & -8.2 \\
\hline
\end{tabular}




\subsubsection{Keyhole parameter set melt pool}

When comparing the melt pool for this parameter set, it is clear that the geometry obtained from the simulation is not close to the experimental results. The width of the melt pool surface only varied by eight per cent from the values obtained experimentally. This is quite an encouraging result. When comparing the depth of the melt pool, the obtained result differed from the experimental mean by $70.4 \%$. However, this value still falls well within two standard deviations of the experimentally obtained results. This is due to the large variation in the melt pool depth due to the instability of the keyhole melting process (Refer to Table III). Although deep penetration into the substrate occurs, which is a characteristic of keyhole mode melting, the melt pool geometry is not representative of the experimental results. It should be noted that no keyhole simulation studies could be found at the time of writing where this melt pool cross-section shape was obtained. This is being investigated further.

\subsection{Effect of unknown and uncertain simulation parameters on melt pool geometry}

During this investigation, it was noted that there were a few key simulation input parameters that were unclear at this stage. Most notably, these include the accommodation coefficient and evaporation pressure coefficients derived from the Clausius Clapeyron equation, and the temperature-dependent absorption of the material. These variables affect the rate of evaporation of the metal, the local pressure above the melt pool caused by evaporation and the energy absorbed by the substrate, respectively. The main reasons for the discrepancies in the results are thought to be the unknown evaporation pressure coefficients and temperaturedependent absorption (Communications, 2021). A study by Ye, et al. (2019) demonstrated that the absorptivity of a Ti6Al4V baseplate without powder can vary between 0.26 and 0.66 depending on the laser power applied to the bed. This finding is significant and needs to be accounted for to ensure accurate simulation in the future. Secondly, there was no known methodology to determine the evaporation pressure coefficients for simulations and it was not clear whether these values changed when different laser power and scanning speeds were applied. Accurate use of evaporation pressure coefficients and absorptivity is critical, as additional simulation work indicated that melt pool dimensions can vary widely by changing only these parameters.

\section{Conclusions}

This study proved that accurate results can be obtained for certain process parameters using numerical modelling. However, the study also clearly demonstrated that accurate results for all process parameters could not be obtained by using an identical simulation setup, verified simulation method, and verified material properties. These inaccuracies currently hinder the possibility of using the software to accurately determine process parameters. The reasons for these inaccuracies are still under investigation. However, certain simulation parameters have been identified that are both unknown, and notably affect melt pool dimensions. A deeper understanding of these parameters, as well as how they can be determined accurately, is required. While the process of characterising melt pool geometry through simulation holds merit, more research is required before its full benefit could be derived.

\section{Acknowledgements}

This project was supported by the Department of Science and Innovation (DSI) under the Collaborative Program in Additive Manufacturing (CPAM) (Contract № CSIR-NLC-CPAM-18MOA-CUT-01). Additional funding was also received from the Central University of Technology, Free State Postgraduate Grant awards.

\section{References}

1. Boivineau, M., Cagran, C., Doytler, D., Eyrand, V., Nadai, M. H. Wilthan, B., \& Pottlacher, G. (2006). Thermophysical Properties of Solid and Liquid Ti-6Al-4V (TA6V) Alloy. International Journal of Thermophysics, 27(2), 507-529.

2. Boley, C. D., Mitchell, S. C., Rubenchik, A. M., \& Wu, S. S. (2016) Metal powder absorptivity: modelling and experiment. Applied Optics 55, 6496-6500. doi:http://dx.doi.org/10.1364/AO.55.006496

3. Brandes, E. A., \& Brook, G. B. (1992). Smithells Metals Reference Book Seventh Edition. Woburn, MA: Butterworth-Heinemann.

4. Cengel, Y. A., \& Ghajar, A. J. (2015). Heat and Mass Transfer Fundamentals \& Applications, Fifth Edition. New York: McGraw-Hill Education.

5. Cheng, B., Loeber, L., Willeck, H., Hartel, U., \& Tuffile, C. (2019) Computational Investigation of Melt Pool Process Dynamics and Pore Formation in Laser Powder Bed Fusion. Journal of Materials Engineering and Performance, 6565-6578. doi:https://doi. org/10.1007/s11665-019-04435-y

6. Egry, I., Holland-Moritz, D., Navokovic, R., Ricci, E., Wunderlich, R., \& Sobezak, N. (2010). Thermophysical Properties of Liquid AlTi-Based Alloys. International Journal of Thermophysics, 949-965.

7. FLOW-3D. (2021, September 09). FLOW-3D AM. Retrieved from FLOW-3D: https://www.flow3d.com/products/flow3d-am/

8. Ford, I. J., \& Lee, T. L. (2001). Entropy production and destruction in models of material evaporation. Journal of Physics D: Applied Physics $34,413-417$.

9. Gibson, I., Rosen, D., \& Stucker, B. (2015). Additive Manufacturing Technologies. New York: Springer.

10. Gong, H., Gu, H., Zeng, K., Dilip, J. J., Pal, D., Stucker, B., Christiansen, D., Beuth, J., Lewandowski, J. J. (2014). Melt Pool Characterization for Selective Laser Melting of Ti-6Al-4V Pre-alloyed Powder. 25th Annual International Solid Freeform Fabrication Symposium, (pp. 256-267).

11. King, W. E., Barth, H. D., Castillo, V. M., Gallegos, G. F., Gibbs, J. W., Hahn, D. E., Kamath, C., Rubenchik, A. M. (2014). Observation of keyhole-mode laser melting in laser powder-bed fusion additive manufacturing. Journal of Materials Processing Technology, Vol. 214 2915-2925.

12. Lu, L., Zhang, S., Xu, J., He, H., \& Zhao, X. (2017). Numerical study of titanium melting by high frequency inductive heating. International Journal of Heat and Mass Transfer, 2021-2028.

13. Majeed, M., Khan, H. M., \& Rasheed, I. (2019). Finite element analysis of melt pool thermal characteristics with passing laser in SLM process. Optik, 194

14. Mills, K. (2002). Recommended Values of Thermophysical Properties for Selected Commercial Alloys. Woodhead Publishing.

15. Milosevic, N., \& Aleksic, I. (2012). Thermophysical properties of solid phase Ti-6Al-4V alloy over a wide temperature range. International Journal of Materials Research, 707-714.

16. Mohr, M., Wunderlich, R., Novakovic, R., Ricci, E., \& Fecht, H. (2020). Precise Measurements of Thermophysical Properties of Liquid Ti-6Al-4V (Ti64) Alloy On Board the International Space Station. Advanced Engineering Materials,22. doi:https://doi.org/10.1002/ adem.202000169

17. Nicoletto, G., Konecna, E., Frka, M., \& Riva, E. (2018). Surface roughness and directional fatigue behaviour of as-built EBM and DMLS Ti6A14V. International Journal of Fatigue 116, 140-148.

18. Rauniyar, S. K., \& Chou, K. (2019). Melt Pool Analysis and Mesoscale Simulation of Laser Powder Bed Fusion Process (L-PBF) with Ti-6Al-4V Powder Particles. JOM, 71 (3), 938-945.

19. Ridolfi, M. R., Folgarait, P., Battaglia, V., Vela, T., Corapi, D., \& Di Schino, A. (2019). Development and calibration of a CFD-based model of the bed fusion SLM additive manufacturing process aimed at optimising laser parameters. Proocedia Structural Integrity, 24, 370-380. 
20. Schmon, A., Aziz, K., \& Pottlacher, G. (2017). Density of liquid Ti-6Al-4V. EPU Web of Conferences, 151. doi:10.1051/ epjconf/201715104003

21. Sun, S., Brandt, M., \& Easton, M. (2017). Powder bed fusion Processes: an overview. In M. Brandt, Laser Additive Manufacturing Materials (pp. 55-77). Duxford: Woodhead Publishing.

22. Tan, P., Shen, F., Li, B., \& Zhou, K. (2019). A thermo-metallurgicalmechanical model for selective laser melting of Ti6Al4V. Materials and Design, 168. doi:10.1016/j.matdes.2019.107642

23. Wu, Y., Hwang, W., San, C., Chang, C., \& Lin, H. (2017). Parametric study of surface morphology for selective laser melting on Ti6Al4V powder bed with numerical and experimental methods. International Journal of Material Forming 11, 807-813. doi:https://doi.org/10.1007/ s12289-017-1391-2
24. Xie, J., Kar, A., Rothenflue, J. A., \& Latham, W. P. (1997) Temperature-dependent absorptivity and cutting capability of $\mathrm{CO} 2$ $\mathrm{Nd}$ :YAG and chemical oxygen-iodine lasers. Journal of Laser Applications 9, 77-85

25. Yadroitsava, I., Els, J., Booysen, G., \& Yadroitsev, I. (2015). Peculiarities of single track formation from Ti6Al4V alloy at different laser power densities by selective laser melting. South African Journal for Industrial Engineering, 26, 86-95.

26. Ye, J., Khairallah, S. A., Rubenchik, A. M., Crumb, M. F., Guss, G., Roehling, J., Belak, J., Matthews, M. J. (2019). Energy Coupling Mechanisms and Scaling Behavior Associated with Laser Powder Bed Fusion Additive Manufacturing. Advanced Engineering Materials, 1900185

27. Communications from several meetings with a CFD engineer at FLOW-3D, latest meeting date Monday, 17 May 2021. 